\title{
ASYMPTOTIC STABILITY FOR ABSTRACT NONLINEAR FUNCTIONAL DIFFERENTIAL EQUATIONS
}

\author{
G. F. WEBB
}

\begin{abstract}
The nonlinear autonomous functional differential equation $\dot{x}(t)$ $=f(x(t))+g\left(x_{t}\right), t \geqslant 0, x_{0}=\phi$ is investigated by means of the theory of semigroups of nonlinear operators. The properties of the semigroup associated with this equation provide stability information about the solutions.
\end{abstract}

1. Introduction. The purpose of this paper is to prove some stability properties of the nonlinear autonomous functional differential equation

(FDE) $\quad \dot{x}(\phi)(t)=f(x(\phi)(t))+g\left(x_{t}(\phi)\right), \quad t \geqslant 0, x_{0}(\phi)=\phi$.

The notation of (FDE) follows $\mathrm{J}$. Hale [4], that is $\phi \in C=C([-r, 0] ; X)$ where $r>0$ and $X$ is a Banach space, $x(\phi)(t):[-r, \infty) \rightarrow X$, and $x_{t}(\phi) \in C$ is defined for each $t \geqslant 0$ by $x_{t}(\phi)(\theta)=x(\phi)(t+\theta), \theta \in[-r, 0]$. In (FDE) we will require a Lipschitz condition on the nonlinear operator $g: C \rightarrow X$ and an accretiveness condition on the nonlinear operator $f: X \rightarrow X$. The ordinary part of (FDE) corresponding to $f$ will act as a damping term for the equation. Our main result can be summarized as follows: Suppose $g$ has Lipschitz constant $\beta$ and $-f+\alpha I$ is accretive. If $\alpha=-\beta$, then (FDE) is stable, and if $\alpha<-\beta$, then (FDE) is asymptotically stable. As a simple example for our problem one can let $X=\mathbf{R}, f(x)=-x^{3}+\alpha x, g(\phi)=h(\phi(-r))$, where $h$ has Lipschitz constant $\beta$, and then (FDE) is the scalar delay equation

$$
\dot{x}(\phi)(t)=-x^{3}(\phi)(t)+\alpha x(\phi)(t)+h(x(\phi)(t-r)) .
$$

Our approach will be to use the general theory of semigroups of nonlinear operators. By allowing $X$ to be a Banach space our results may be applied to partial functional differential equations as in [7]. For related treatments of our problem one should see [7]-[9].

2. Definitions. For an arbitrary Banach space $Y$, a nonlinear operator $A$ from $Y$ to $Y$ is accretive provided

$$
\|(I+\lambda A) x-(I+\lambda A) y\| \geqslant\|x-y\| \text { for all } x, y \in D(A), \lambda>0 .
$$

Our results rely upon the following general theorem, due to $\mathrm{M}$. Crandall and T. Liggett [1], from nonlinear semigroup theory: Suppose for some $\gamma \in \mathbf{R}$,

Received by the editors December 26, 1974.

AMS (MOS) subject classifications (1970). Primary 34K20; Secondary 47H15.

Key words and phrases. Autonomous functional differential equation, stability, asymptotic stability, nonlinear accretive operator, nonlinear semigroup of operators. 
$A+\gamma I: Y \rightarrow Y$ is accretive and $R(I+\lambda A)$ is onto for all sufficiently small $\lambda>0$. Then,

$$
\lim _{n \rightarrow \infty}(I+t / n A)^{-n} x \stackrel{\text { def }}{=} T(t) x \text { exists for } x \in \overline{D(A)}, t \geqslant 0 .
$$

Moreover, $T(t), t \geqslant 0$, is a strongly continuous semigroup of nonlinear operators on $\overline{D(A)}$, that is,

$$
T(0) x=x \quad \text { for all } x \in \overline{D(A)}
$$

$T(t) x$ is continuous in $t$ for each fixed $x \in \overline{D(A)}$;

$$
\begin{gathered}
T\left(t_{1}+t_{2}\right)=T\left(t_{1}\right) T\left(t_{2}\right) \quad \text { for } t_{1}, t_{2} \geqslant 0 ; \\
\|T(t) x-T(t) y\| \leqslant e^{\gamma t}\|x-y\| \quad \text { for } x, y \in \overline{D(A)}, t \geqslant 0 .
\end{gathered}
$$

Let $C_{0}$ be the subspace of $C$ given by $C_{0}=\{\phi \in C: \phi(0)=0\}$. Define the linear operators

$$
\begin{gathered}
A_{0}: C_{0} \rightarrow C_{0} \quad \text { by } A_{0} \phi=-\phi^{\prime}, \quad D\left(A_{0}\right)=\left\{\phi \in C_{0}: \phi^{\prime} \in C_{0}\right\}, \\
A_{1}: C \rightarrow C \quad \text { by } A_{1} \phi=-\phi^{\prime}, \quad D\left(A_{1}\right)=\left\{\phi \in C_{0}: \phi^{\prime} \in C\right\} .
\end{gathered}
$$

From the theory of semigroups of linear operators one has that $A_{0}$ is accretive in $C_{0}, R\left(I+\lambda A_{0}\right)=C_{0}$ for $\lambda>0, D\left(A_{0}\right)$ is dense in $C_{0}, A_{0}$ is the infinitesimal generator of a strongly continuous semigroup of linear contractions on $C_{0}$, and

$$
\lim _{\lambda \rightarrow 0^{+}}\left(I+\lambda A_{0}\right)^{-1} \phi=\phi \quad \text { for all } \phi \in C_{0} .
$$

Also, $A_{1}$ is accretive in $C, R\left(I+\lambda A_{1}\right)=C$ for $\lambda>0$, but $D\left(A_{1}\right)$ is not dense in $C$. If $\lambda>0$ then

$(2.10)\left(\left(I+\lambda A_{0}\right)^{-1} \phi\right)(\theta)=\left(\frac{e^{\theta / \lambda}}{\lambda}\right) \int_{\theta}^{0} e^{-s / \lambda} \phi(s) d s, \quad \phi \in C_{0}, \theta \in[-r, 0]$

$$
\begin{aligned}
& \left(\left(I+\lambda A_{1}\right)^{-1} \phi\right)(\theta)=\left(\frac{e^{\theta / \lambda}}{\lambda}\right) \int_{\theta}^{0} e^{-s / \lambda} \phi(s) d s, \quad \phi \in C, \theta \in[-r, 0] \\
& \left(I+\lambda A_{1}\right)^{-1} \phi=\left(I+\lambda A_{0}\right)^{-1}(\phi-\phi(0))+\left(1-e^{\theta / \lambda}\right) \phi(0), \quad \phi \in C .
\end{aligned}
$$

3. The nonlinear semigroup for (FDE). In this section we shall construct a nonlinear semigroup which can be associated with (FDE) by exhibiting its generator in the sense of (2.2). In what follows we shall suppose that for some $\alpha \in \mathbf{R}, f: X \rightarrow X$ such that $-f+\alpha I$ is accretive and $R(I-\lambda f)=X$ for $0<\lambda<1 / \max \{0, \alpha\}$. From (2.1) this implies that for $x, y \in D(f), \lambda>0$,

$$
\left\|(I-\lambda f)^{-1} x-(I-\lambda f)^{-1} y\right\| \leqslant(1 /(1-\lambda \alpha))\|x-y\|,
$$


and

(3.2) $\lim _{\lambda \rightarrow 0^{+}}(I-\lambda f)^{-1} x=x \quad$ for $x \in \overline{D(f)} \quad$ (see [1, Lemma 1.2(ii)]).

We shall also suppose that $g: C \rightarrow X$ is Lipschitz continuous with Lipschitz constant $\beta$. Define $A: C \rightarrow C$ by $A \phi=-\phi^{\prime}$ with $D(A)=\left\{\phi \in C: \phi^{\prime} \in C\right.$, $\left.\phi(0) \in D(f), \phi^{\prime-}(0)=f(\phi(0))+g(\phi)\right\}$.

Proposition 1. $A+\gamma I$ is accretive in $C$ and $R(I+\lambda A)=C$ for $0<\lambda$ $<1 / \gamma$, where $\gamma=\max \{0, \alpha+\beta\}$.

Proof. We first show $I+\lambda A$ is $1-1$ and onto. Let $0<\lambda<1 / \gamma, \psi \in C$. Define the mapping $k: X \rightarrow X$ by

$$
k(b)=(I-\lambda f)^{-1}\left(\psi(0)+\lambda g\left(e^{\theta / \lambda} b+\left(I+\lambda A_{1}\right)^{-1} \psi\right)\right), \quad b \in X .
$$

Then $k$ is a strict contraction on $X$, since $\left\|k\left(b_{1}\right)-k\left(b_{2}\right)\right\| \leqslant(\lambda \beta /(1-\lambda \alpha))$ - $\left\|b_{1}-b_{2}\right\|$. Thus, $k$ has a unique fixed point $b_{0}$, and

$$
\phi(\theta) \stackrel{\text { def }}{=} e^{\theta / \lambda} b_{0}+\left(\left(I+\lambda A_{1}\right)^{-1} \psi\right)(\theta)
$$

solves uniquely $\phi-\lambda \phi^{\prime}=\psi, \phi^{\prime-}(0)=f(\phi(0))+g(\phi)$, that is, $(I+\lambda A) \phi$ $=\psi$.

Next, we will show that for all $\psi_{1}, \psi_{2} \in C, 0<\lambda<1 / \gamma$,

$$
\left\|(I+\lambda A)^{-1} \psi_{1}-(I+\lambda A)^{-1} \psi_{2}\right\| \leqslant(1 /(1-\lambda \gamma))\left\|\psi_{1}-\psi_{2}\right\| .
$$

Let $(I+\lambda A) \phi_{1}=\psi_{1},(I+\lambda A) \phi_{2}=\psi_{2}$, and $\theta \in[-r, 0]$ such that $\| \phi_{1}(\theta)$ - $\phi_{2}(\theta)\|=\| \phi_{1}-\phi_{2} \|$. From (3.3),(3.4), and (2.11) we have

$$
\begin{aligned}
\left\|\phi_{1}-\phi_{2}\right\|= & \left\|\phi_{1}(\theta)-\phi_{2}(\theta)\right\| \\
\leqslant & \left(e^{\theta / \lambda} /(1-\lambda \alpha)\right)\left(\left\|\psi_{1}(0)-\psi_{2}(0)\right\|+\lambda \beta\left\|\phi_{1}-\phi_{2}\right\|\right) \\
& +\left(1-e^{\theta / \lambda}\right)\left\|\psi_{1}-\psi_{2}\right\|
\end{aligned}
$$

which implies

$$
\left\|\phi_{1}-\phi_{2}\right\| \leqslant\left(\left(1-\lambda \alpha+\lambda \alpha e^{\theta / \lambda}\right) /\left(1-\lambda \alpha-\lambda \beta e^{\theta / \lambda}\right)\right)\left\|\psi_{1}-\psi_{2}\right\| .
$$

If $0 \leqslant \alpha+\beta$, then

$$
\left(1-\lambda \alpha+\lambda \alpha e^{\theta / \lambda}\right) /\left(1-\lambda \alpha-\lambda \beta e^{\theta / \lambda}\right) \leqslant 1 /(1-\lambda(\alpha+\beta))=1 /(1-\lambda \gamma) .
$$

If $\alpha+\beta \leqslant 0$, then

$$
\begin{aligned}
\left(1-\lambda \alpha+\lambda \alpha e^{\theta / \lambda}\right) /(1 & \left.-\lambda \alpha-\lambda \beta e^{\theta / \lambda}\right) \\
& \leqslant\left(1-\lambda \alpha-\lambda \beta e^{\theta / \lambda}\right) /\left(1-\lambda \alpha-\lambda \beta e^{\theta / \lambda}\right)=1 /(1-\lambda \gamma) .
\end{aligned}
$$

In either case, (3.5) holds, and this yields the accretiveness of $A+\gamma I$.

Proposition 2. $\overline{D(A)}=\{\psi \in C: \psi(0) \in \overline{D(f)}\}$.

Proof. Let $(I+\lambda A) \phi=\psi$ as in Proposition 1. Using (3.3) and (3.4) we see 
that

$$
\begin{aligned}
\|\phi(0)-\psi(0)\|= & \left\|(I-\lambda f)^{-1}(\psi(0)+\lambda g(\phi))-\psi(0)\right\| \\
\leqslant & (\lambda /(1-\lambda \alpha))(\beta\|\phi-\psi\|+\|g(\psi)\|) \\
& +\left\|(I-\lambda f)^{-1} \psi(0)-\psi(0)\right\| .
\end{aligned}
$$

Also, from (2.12),

$$
\|\phi-\psi\| \leqslant\left\|\left(I-\lambda A_{0}\right)^{-1}(\psi-\psi(0))-(\psi-\psi(0))\right\|+\|\phi(0)-\psi(0)\| .
$$

From (3.6) and (3.7) we obtain

$$
\begin{aligned}
\|\phi-\psi\| \leqslant & ((1-\lambda \alpha) /(1-\lambda(\alpha+\beta))) \\
& \cdot\left(\left\|\left(I-\lambda A_{0}\right)^{-1}(\psi-\psi(0))-(\psi-\psi(0))\right\|\right. \\
& \left.\quad+\left\|(I-\lambda f)^{-1} \psi(0)-\psi(0)\right\|+(\lambda /(1-\lambda \alpha))\|g(\psi)\|\right) .
\end{aligned}
$$

From the general theory of accretive operators (see [1, Lemma 1.2 (ii)]) we have from Proposition 1 that

$$
\overline{D(A)}=\left\{\psi \in C: \lim _{\lambda \rightarrow 0^{+}}(I+\lambda A)^{-1} \psi=\psi\right\} .
$$

Then, (2.9), (3.2), (3.8), and (3.9) imply the conclusion.

By virtue of Propositions 1 and 2 we may use formula (2.2) to define a nonlinear semigroup $T(t), t \geqslant 0$, on $\overline{D(A)}$ with generator $A$. If $\overline{D(f)}=X$, then $\overline{D(A)}=C$. If $f$ is linear and densely defined in $X$, then $-A$ is exactly the infinitesimal generator of $T(t), t \geqslant 0$ (see [7] or [8]).

The question arises as to when the semigroup $T(t), t \geqslant 0$, gives solutions to (FDE). One can use the methods of H. Flaschka and M. Leitman [3] to show that $T(t), t \geqslant 0$, always has the following property: If for each $\phi \in \overline{D(A)}$ we define

$$
\begin{aligned}
x(\phi)(t) & =\phi(t) \text { for }-r \leqslant t \leqslant 0, \\
& =(T(t) \phi)(0) \quad \text { for } t \geqslant 0 ;
\end{aligned}
$$

then $T(t) \phi=x_{t}(\phi)$. In the case that $f$ is everywhere defined and continuous the methods of [3] can be used to show that the function $x(\phi)(t)$ in (3.10) satisfies (FDE) for all $\phi \in D(A)$. The proof of these facts carries over essentially without change from [3]. In the case that $X$ is a Hilbert space we can show the following.

Proposition 3. If $X$ is a Hilbert space, then for all $\phi \in D(A)$ the function $x(\phi)(t)$ in $(3.10)$ satisfies

$$
\dot{x}(\phi)(t)=f(x(\phi)(t))+g\left(x_{t}(\phi)\right) \text { for a.a. } t \geqslant 0, x_{0}(\phi)=\phi .
$$

Proof. We will use the notation and results from [1]. Let $\phi \in D(A)$. We show first that for all $h \in X, t \geqslant 0$, 


$$
\left.\langle(T(t) \phi)(0), h\rangle=\langle\phi(0), h\rangle+\int_{0}^{t}\langle(f(T(s) \phi)(0))+g(T(s) \phi)) d s, h\right\rangle .
$$

As in [3] we have that

$$
\left(T^{\lambda}(t) \phi\right)(0)=\phi(0)+\int_{0}^{t}\left(f\left(\left(J_{\lambda} T^{\lambda}(s) \phi\right)(0)\right)+g\left(J_{\lambda} T^{\lambda}(s) \phi\right)\right) d s,
$$

and $J_{\lambda} T^{\lambda}(s) \phi$ converges strongly to $T(s) \phi$ as $\lambda \rightarrow 0$. By virtue of the Lebesgue dominated convergence theorem, to establish (3.12) it suffices to show that $f\left(\left(J_{\lambda} T^{\lambda}(s) \phi\right)(0)\right)$ is bounded in $\lambda$ and converges weakly to $f((T(s) \phi)(0))$ as $\lambda \rightarrow 0$. The boundedness follows from the Lipschitz continuity of $g$, the fact that $\phi \in D(A)$, and the inequality

$$
\text { const } \begin{aligned}
\|A \phi\| & \geqslant\left\|A J_{\lambda} T^{\lambda}(s) \phi\right\| \geqslant\left\|\left(A J_{\lambda} T^{\lambda}(s) \phi\right)(0)\right\| \\
& =\left\|f\left(\left(J_{\lambda} T^{\lambda}(s) \phi\right)(0)\right)+g\left(J_{\lambda} T^{\lambda}(s) \phi\right)\right\| .
\end{aligned}
$$

The weak convergence follows from the fact that $f$ is maximal accretive in $X$ by a well-known argument of accretive operator theory (see [2] or [6]). Then (3.12) implies that $x(\phi)(t)$ is weakly differentiable for almost all $t \geqslant 0$. Also, since $\phi \in D(A), T(t) \phi$ is Lipschitz continuous in $t$ (see (1.11) of [1]), and therefore $x(\phi)(t)$ is strongly of bounded variation in $t$. By [5, Theorem 3.8.6, p. 88], $x(\phi)(t)$ is strongly differentiable almost everywhere in $t$ and (3.11) holds.

Now we consider the stability properties of (FDE). If $\alpha=-\beta$, then the trajectories of $T(t), t \geqslant 0$, are stable in the sense of (2.6) with $\gamma=0$. If $\alpha<-\beta$, the proposition below yields the asymptotic stability of (FDE) if $X$ is a Hilbert space.

Proposition 4. Suppose $\alpha<-\beta$ and $X$ is a Hilbert space. Then $T(t), t \geqslant 0$, is asymptotically stable in the sense that there exists a unique point $\psi_{0} \in C$ such that $\lim _{t \rightarrow \infty} T(t) \psi=\psi_{0}$ for all $\psi \in C$. Moreover, $\psi_{0}$ is a constant function in $C$.

Proof. Define $j: X \rightarrow X$ by $j(b)=(I-f)^{-1}(b+g(b \cdot \mathbf{1}))$, where $b \in X$ and 1 denotes the constant function identically 1 on $[-r, 0]$. Since $\alpha+\beta<0$, $j$ is a strict contraction with Lipschitz constant $\leqslant(1+\beta) /(1-\alpha)$. Let $b_{0}$ be the unique fixed point of $j$ in $X$ and define $\psi_{0}=b_{0} \cdot 1$. Then $\psi_{0} \in D(A)$ and $A \psi_{0}=0$. From formula (2.2) we have that $T(t) \psi_{0}=\psi_{0}$ for all $t \geqslant 0$.

To prove the conclusion it suffices to show that

$$
\lim _{t \rightarrow \infty} \sup _{\phi, \psi \in C}\|T(t) \phi-T(t) \psi\| /\|\phi-\psi\|=0 .
$$

Let $\phi, \psi \in D(A)$ and let $x(t)$ and $y(t)$ solve (3.11) for $\phi$ and $\psi$, respectively. Then, for almost all $t \geqslant 0$,

$$
\begin{aligned}
\frac{1}{2}(d / d t)\|x(t)-y(t)\|^{2} & =\left\langle f(x(t))+g\left(x_{t}\right)-f(y(t))-g\left(y_{t}\right), x(t)-y(t)\right\rangle \\
& \leqslant \alpha\|x(t)-y(t)\|^{2}+\beta\|T(t) \phi-T(t) \psi\|\|x(t)-y(t)\| \\
& \leqslant \alpha\|x(t)-y(t)\|^{2}+\beta\|\phi-\psi\|^{2} .
\end{aligned}
$$

By Gronwall's inequality, (3.14) implies that for $t \geqslant 0$, 


$$
\|x(t)-y(t)\| \leqslant\|\phi-\psi\|\left(\beta /-\alpha-e^{2 \alpha t}(1+\beta /-\alpha)\right)^{1 / 2} .
$$

Then (3.15) implies that for $t \geqslant 0$,

$$
\|T(t) \phi-T(t) \psi\| \leqslant\|\phi-\psi\|\left(\beta /-\alpha-e^{2 \alpha(t-r)}(1+\beta /-\alpha)\right)^{1 / 2} .
$$

Using (2.6) with $\gamma=0$, we have that (3.16) holds for all $\phi, \psi \in \overline{D(A)}$. But this means that $T(t)$ is a strict contraction when $t>r$ and this fact, together with (2.5), yields (3.13).

In conclusion we remark that all of our propositions carry over easily to the case that $f$ is a multivalued accretive operator, a class of nonlinear operators that is very general and extensively developed (see, e.g., [1] and [2]). Also, Propositions 3 and 4 carry over readily to the case that $X$ is a uniformly convex Banach space.

\section{REFERENCES}

1. M. G. Crandall and T. M. Liggett, Generation of semi-groups of nonlinear transformations on general Banach spaces, Amer. J. Math. 93 (1971), 265-298. MR 44 \#4563.

2. M. G. Crandall and A. Pazy, Semi-groups of nonlinear contractions and dissipative sets, J. Functional Analysis 3 (1969), 376-418. MR 39 \#4705.

3. H. Flaschka and M. Leitman, On semigroups of non-linear operators and the solution of the functional differential equation $\dot{x}(t),=F\left(x_{t}\right)$, J. Math. Anal. Appl. 49 (1975), 649-658.

4. J. Hale, Functional differential equations, Appl. Math. Series, vol. 3, Springer-Verlag, New York, 1971.

5. E. Hille and R. S. Phillips, Functional analysis and semi-groups, rev. ed., Amer. Math. Soc. Colloq. Publ., vol. 31, Amer. Math. Soc., Providence, R.I., 1957. MR 19, 664.

6. T. Kato, Nonlinear semigroups and evolution equations, J. Math. Soc. Japan 19 (1967), 508520. MR 37 \# 1820.

7. C. Travis and G. Webb, Existence and stability for partial functional differential equations, Trans. Amer. Math. Soc. 200 (1974), 395-418.

8. G. Webb, Autonomous nonlinear functional differential equations and nonlinear semigroups, $\mathrm{J}$. Math. Anal. Appl. 46 (1974), 1-12.

9. $\quad$ Functional differential equations and nonlinear semigroups in $L^{p}$-spaces, J. Differential Equations (to appear).

Department of Mathematics, Vanderbilt University, Nashville, Tennessee 37235

Instituto Matematico, Universita di Roma, 00100 Roma, Italia 